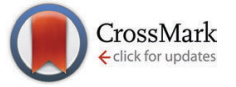

Cite this: Phys. Chem. Chem. Phys., 2015, 17, 11110

DOI: $10.1039 / \mathrm{c} 5 \mathrm{cp} 90049 \mathrm{~b}$

www.rsc.org/pccp

\section{Correction: Scaling properties of information- theoretic quantities in density functional reactivity theory}

\author{
Chunying Rong, ${ }^{\text {ab }}$ Tian Lu, $^{c}$ Paul W. Ayers, ${ }^{\text {b }}$ Pratim K. Chattaraj $^{\mathrm{d}}$ and Shubin Liu*ae
}

Correction for 'Scaling properties of information-theoretic quantities in density functional reactivity theory' by Chunying Rong et al., Phys. Chem. Chem. Phys., 2015, 17, 4977-4988.

The authors would like to amend the text by the following four points:

1. Eqn (19) of the text is in error. The correct formula is as follows:

$$
S_{\sigma}=\frac{S_{\mathrm{S}}}{N}+\ln N
$$

2. Because of (1), numerical results of $S_{\sigma}$ in Tables 1 and 2 (column 4) are incorrect. The correct results are shown in Table C1 below, where all data are found to be positive.

3. The correct correlation coefficient $\left(R^{2}\right)$ and root-mean-square deviation (RMSD) values for $S_{\sigma}$ from the least-square-fitting in Table 3 are shown in Table C2.

4. Correlation results of $S_{\sigma}$ at atoms, molecules and atoms-in-molecules levels shown in Fig. 1a, 2a, 3a, 5a, and 6a are invalidated and should be replaced by the results in Table C2. However, as can be seen from the table, strong correlations at the atoms-in-molecules level are still observed.

\footnotetext{
${ }^{a}$ Key Laboratory of Chemical Biology and Traditional Chinese Medicine Research (Ministry of Education of China), College of Chemistry and Chemical Engineering, Hunan Normal University, Changsha, Hunan 410081, China

${ }^{b}$ Department of Chemistry, McMaster University, Hamilton, Ontario L8S 4M1, Canada. E-mail: ayers@mcmaster.ca

${ }^{c}$ Beijing Kein Research Center for Natural Sciences, Beijing 100022, P. R. China

${ }^{d}$ Department of Chemistry and Center for Theoretical Studies, Indian Institute of Technology, Kharagpur 721302, India

${ }^{e}$ Research Computing Center, University of North Carolina, Chapel Hill, North Carolina 27599-3420, USA. E-mail: shubin@email.unc.edu
} 
Table C1 Shannon entropy with the shape function for 36 neutral atoms and 42 molecular systems. Atomic units

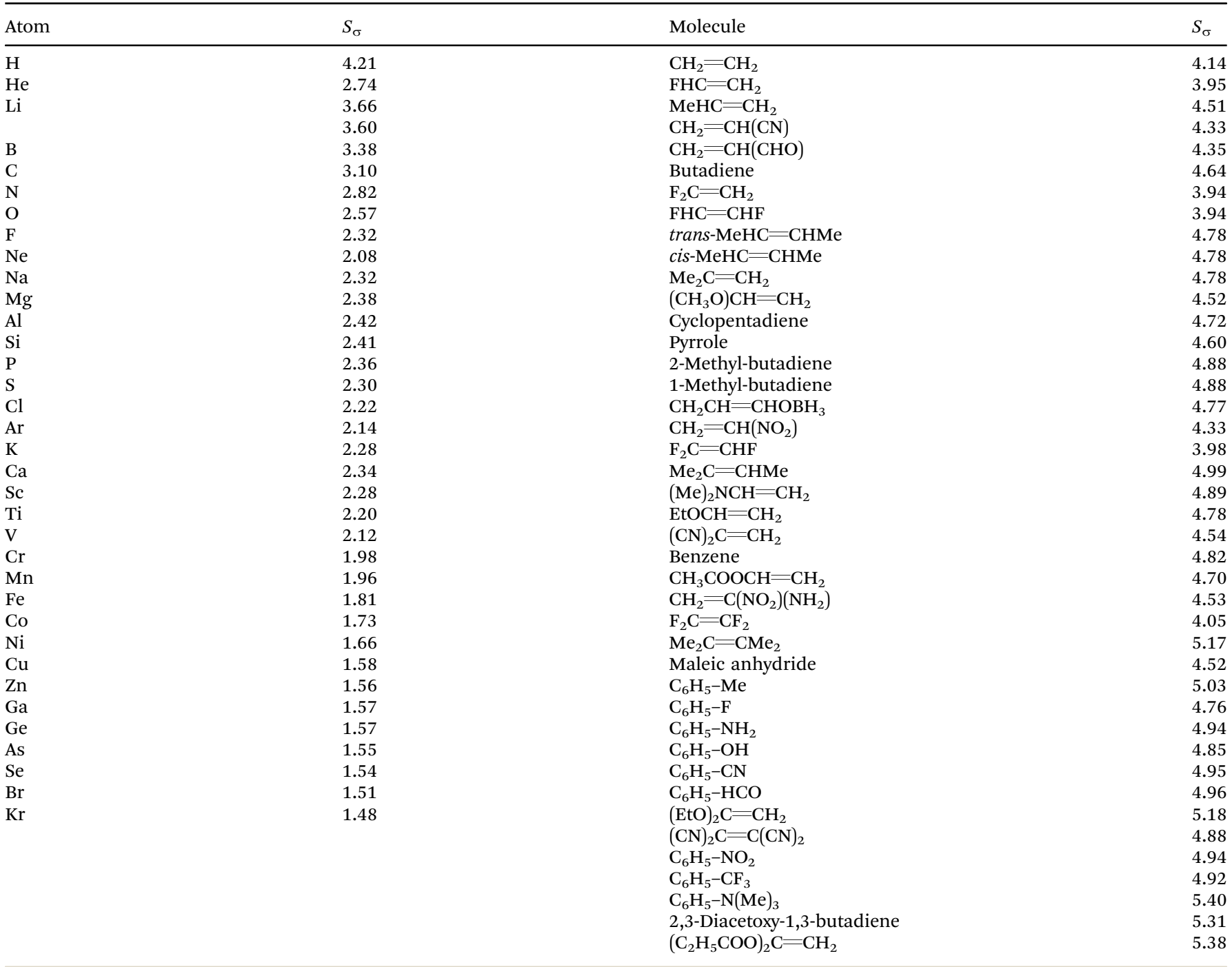

Table C2 The correlation coefficient $R^{2}$ and root-mean-square deviation (RMSD) values for Shannon entropy with the shape function, $S_{\sigma}$, at atomic, molecular and atoms-in-molecules levels with AIM, Becke, and Hirshfeld partitioning schemes

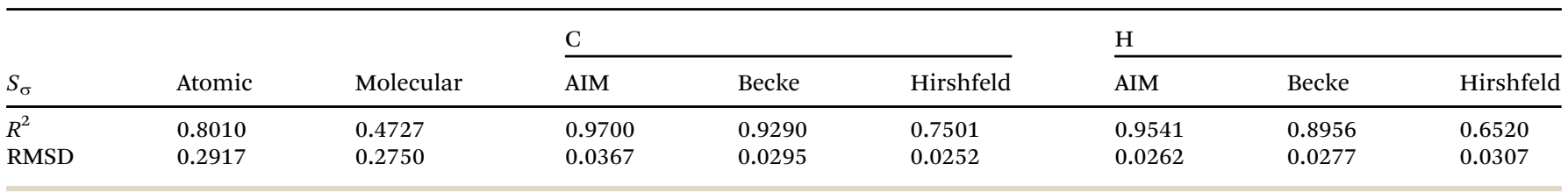

The Royal Society of Chemistry apologises for these errors and any consequent inconvenience to authors and readers. 